\title{
Lettres à la rédaction
}

\author{
Monsieur le Rédacteur en chef,
}

Suite à la réunion de formation médicale continue que la section «Rayonnements Non Ionisants » (RNI) de la SFRP a organisée le 6 octobre dernier à Grenoble en collaboration avec l'Observatoire régional de la santé Rhône-Alpes, il semble important de porter à la connaissance de vos lecteurs notre réflexion concernant l'information relative aux effets éventuels des ondes électromagnétiques sur la santé et la nécessité de continuer à programmer de telles séances de formation au profit des professionnels de santé. À ce jour, aucune donnée scientifique ne permet d'envisager une remise en cause des recommandations faites par l'International Commission of Non Ionizing Radiation Protection (ICNIRP, 1998).

L'information à destination de la population est véhiculée essentiellement par les médias et les associations de défense des citoyens, voire par les associations via les médias. La recherche du sensationnel et du scoop pour faire vendre un journal ou monter l'audimat introduit de toute évidence un biais dans le choix des nouvelles à transmettre. Nombre d'informations alarmistes sont consacrées au risque lié à ces expositions, dans la presse écrite et audio-visuelle, sur Internet, voire dans des revues d'informations médicales où la notion de danger est mise en avant. Des «conclusions» sont établies à partir de productions scientifiques isolées, publiées ou non, souvent sorties de leur contexte, ou bien à partir de cas particuliers jouant sur l'émotion. L'ensemble est traité de façon pseudo-scientifique pour servir des thèses préétablies. Une méconnaissance du sujet, des raccourcis fâcheux, l'amalgame avec les risques du tabac ou de l'amiante sont fréquents et rendent discutable une grosse part de l'information due au public.

Pour couronner le tout, des chercheurs contribuent eux-mêmes à la confusion et à la montée de l'inquiétude. Certains se laissent tenter par des extrapolations de leurs résultats qui leur valent une notoriété instantanée ou encore sont poussés à tirer des conclusions avant même la fin des travaux quand ils ne font pas tout simplement l'impasse sur la dosimétrie.

Les résultats des études peuvent aussi générer une anxiété significative. Nous pouvons prendre comme exemple l'épidémiologie et la désormais fameuse étude INTERPHONE, dimensionnée pour avoir une puissance statistique suffisante avec les données de treize pays (mais disséminée par morceaux), grevée par le fait que chaque équipe a voulu publier ses propres résultats avant que l'ensemble des résultats ne soit analysé. Ceci fait les choux gras de la presse et des informateurs alarmistes qui en rajoutent en laissant supposer qu'il s'agit de «fuites» ou de conclusions intermédiaires, que les chercheurs n'arrivent pas à se mettre d'accord ou ne peuvent publier sous la pression des lobbies industriels... Il n'y a pas de fumée sans feu!

Par ailleurs, on assiste à des initiatives conduites par des experts autoproclamés n'hésitant pas à utiliser leur « autorité médicale » ou à se draper dans la cape du lanceur d'alerte à grand

DOI: $10.1051 /$ radiopro/2009003

RADIOPROTECTION - VOL. 44 - N 1 (2009)

Article published by EDP Sciences and available at http://www.radioprotection.org 
renfort de medias. Les médias font également la part belle aux contre-experts indépendants d'origines diverses aux titres ronflants qui sont bien plus populaires que les experts qui travaillent effectivement sur le sujet, l'indépendance étant devenue le gage de sérieux. Le message, même s'il est affolant est en tout cas bien reçu car le doute propre au scientifique y est balayé d'un revers de manche au profit d'affirmations claires et nettes.

Bref, l'incertitude est anxiogène et beaucoup d'éléments d'informations disparates concourent à inquiéter la population. Les personnes les plus sceptiques finissent par se dire qu'il doit peut-être y avoir un risque pour que cela fasse tant de bruit, d'autant que les théories du complot ont actuellement bonne presse. D'autres adhèrent tout de go aux élucubrations des militants et peuvent développer des pathologies, par contre bien réelles, attribuées à une hypersensibilité aux ondes électromagnétiques. Les études réalisées à ce jour, en double aveugle, concluent à une absence de relation causale entre les symptômes observés et l'exposition au champ électromagnétique. Dans le meilleur des cas, la personne souffrante va se tourner vers son médecin traitant pour obtenir des précisions sur ce risque ou chercher celui qui voudra bien confirmer que sa maladie est due à l'exposition aux champs électromagnétiques. "J'ai vu à la télé que... Qu'est ce que vous en pensez docteur ? ». Souvent, le médecin sera bien en peine de répondre car il dispose, ni plus ni moins, de la même information que son patient. Au mieux, avouera t'il son ignorance et sa volonté de s'informer de façon objective (mais où ?), au pire, il reprendra le message des médias.

Quid de l'information scientifique ? Elle est par nature non sensationnelle. Toutefois, il existe une littérature abondante sur le sujet. De nombreux rapports d'expertise scientifique sont disponibles en ligne (JOCE, 1999 ; European Commission, 2007 ; WHO, 2007 ; MTHR, 2007) et même en français (CSHPF, 2005 ; AFSSE, 2005 ; Supélec, 2006 ; INC, 2008a, 2008b). Ils sont pratiquement tous dévoyés et jugés « corrompus » par les associations qui ne jurent que par le rapport BioInitiative dont le poids et l'impact réside plus dans son volume considérable que dans son contenu fort discutable.

En conclusion, le sujet est particulièrement complexe. Médecins et scientifiques non familiers de l'interdisciplinarité inhérente au sujet ont de quoi y perdre leur latin. Mais le corps médical doit être en mesure de faire la part des choses. Nous ne sommes pas là pour clamer il n'y a rien et il n'y aura jamais rien car la science « procède par rature ». On peut simplement retenir qu'aucun risque n'a encore été mis en évidence et les recherches se poursuivent. Tenons nous en aux faits, aux effets scientifiquement avérés et reproductibles. C'est aux politiques de décider quelle attitude adopter face à l'absence de certitude et aux inquiétudes des citoyens. C'est ce qui justifie actuellement la mise en œuvre du principe de précaution et d'autres initiatives variables selon les communes.

Il est urgent, maintenant, que les connaissances scientifiques soient transmises autrement que dans des revues scientifiques, de haut niveau certes, mais incompréhensibles pour les non spécialistes, en particulier à destination des médecins qui reçoivent en première ligne les questions posées par leurs patients. C'est pourquoi il nous semble important que la section RNI de la SFRP continue à programmer de telles séances de formation au profit des professionnels de santé.

Dr Martine Souques, Présidente de la section RNI

Dr Anne Perrin, Vice-présidente de la section RNI 


\section{RÉFÉRENCES}

AFSSE (2005) Téléphonie mobile \& santé, Agence française de sécurité sanitaire environnementale, Maisons Alfort.

http://www.afsset.fr/upload/bibliotheque/184632204692143805342647948037/telephonie_mobile_2005.pdf

CSHPF (2005) Conseil supérieur d'hygiène publique de France, avis relatif aux champs magnétiques d'extrêmement basse fréquence, Paris.

http://www.sante.gouv.fr/htm/dossiers/cshpf/a_mv_030305_champs_ebf.pdf

European Commission (2007) Scientific Committee on Emerging and Newly Identified Health Risks, Possible effects of Electromagnetic Fields (EMF) on Human Health, Bruxelles.

http://ec.europa.eu/health/opinions2/fr/champs-electromagnetiques/index.htm

ICNIRP (1998) Guidelines for limiting exposure to time-varying electric, magnetic and electromagnetic fields (up to $300 \mathrm{GHz}$ ), Health Phys. 74, 494-522.

http://www.icnirp.de/documents/emfgdl.pdf

INC (2008a) Téléphone portable et risque de cancer : l'état des connaissances, Institut national du cancer, Paris (juin 2008).

http://www.e-cancer.fr/v1/index2.php?option=com_redaction\&do_pdf=1\&id=1988\&lang=1 \&vers $=1$

INC (2008b) Téléphones portables et santé : que savons-nous? Institut national du cancer, Paris. http://www.e-cancer.fr/v1/index2.php?option=com_redaction\&do_pdf=1\&id=1996\&lang=1 \&vers=1

JOCE (1999) Conseil des Communautés européennes, recommandation du Conseil du 12 juillet 1999 relative à la limitation du public aux champs électromagnétiques (de $0 \mathrm{~Hz}$ à $300 \mathrm{GHz}$ ), Journal officiel des Communautés européennes L199, 59-70.

http://eur-lex.europa.eu/LexUriServ/LexUriServ.do?uri=OJ:L:1999:199:0059:0070:FR:PDF

MTHR (2007) Mobile communication and health research program. Report 2007. Health Protection Agency, Chilton, Didcot, UK.

http://www.mthr.org.uk/documents/MTHR_report_2007.pdf

Supélec (2006) Étude "RLAN et champs électromagnétiques »-Synthèse des études conduites par Supélec, Gif-sur-Yvette.

http://www.arcep.fr/uploads/tx_gspublication/synth-etudesupelec-wifi-dec06.pdf

WHO (2007) Extremely low frequency fields, Environmental health criteria 238. World health organization, Geneva.

http://www.who.int/mediacentre/factsheets/fs205/fr/ 


\section{LETTRES À LA RÉDACTION}

\section{Monsieur le Président,}

Je souhaiterais faire un appel aux formateurs professionnels accueillant des stagiaires.

La radioprotection, discipline à caractère essentiellement technique ou scientifique subira très probablement dans les années à venir, comme c'est déjà le cas dans la majorité des domaines de ce type, certaines difficultés de recrutement. On ne cache plus qu'il existe un malaise général du système éducatif et notre revue n'est pas l'endroit prévu pour chercher des causes (manque de sélection, « secondarisation » de l'enseignement supérieur, laxisme de l'enseignement secondaire...). Tout le monde est conscient que des réformes s'imposent. Leurs mises en place s'annoncent difficiles car, sur le terrain, certains établissements jouent clairement la carte de la quantité contre la qualité. Cette tendance se trouve malheureusement accentuée par quelques décideurs locaux, noyant la réalité et les vrais besoins des formations dans des statistiques douteuses basées sur des considérations qui feraient couler n'importe quelle autre entreprise.

Le rôle des formateurs professionnels encadrant les stages pratiques de fin d'études devient ainsi crucial pour le maintien de la qualité des diplômes. La marge de manœuvre par rapport à toute sorte de sélection par les équipes pédagogiques sur place se réduit d'une année à l'autre. La collaboration entre les équipes d'accueil professionnelles et les équipes pédagogiques doit se renforcer et devient incontournable. Accueillir un stagiaire médiocre ne signifie pas que la formation en question soit médiocre. Cependant, la formation risque d'être perçue comme telle. Il peut être recommandé par exemple de solliciter avant la signature de la convention de stage les relevés de notes ou l'appréciation générale auprès des responsables de formation pour éviter des " mauvaises surprises », sans pour autant défavoriser les étudiants bons et motivés. La notation et l'évaluation du stagiaire doivent obligatoirement correspondre à son travail et à sa vraie valeur car elles deviennent de plus en plus souvent, grâce au poids des coefficients affectés à la note de stage, le « Sésame » pour l'obtention du diplôme. Toutes sur-notations ou sous-notations posent par la suite des problèmes aux jurys pour ne pas pénaliser soit la qualité des formations, soit les candidats. Heureusement en pratique la grande majorité des professionnels jouent le jeu et les dérives au niveau des évaluations sont relativement rares.

Ainsi, il faut appliquer une grande vigilance et une coopération efficace des formateurs de tous les niveaux pour garantir dans l'avenir la qualité des métiers à responsabilité.

Pr. L. Makovicka

Enseignant-chercheur

Université de Franche-Comté, Montbéliard. 